\title{
LAS INTERACCIONES ENTRE LOS PAIISES DEL AFTA Y DE LA APEC Y SUS INFLUENCIAS EN LA REGIÓN
}

\author{
Teófilo C. Daquila \\ UNIVERSIDAD NACIONAL DE SINGAPUR
}

$\mathrm{E}$ n la cumbre de Seattle de 1993 , los líderes de la APEC emitieron una declaración y acordaron trabajar en tres grandes áreas: la liberalización del comercio, la facilitación del comercio y la cooperación económica. La liberalización del comercio y la inversión implica eliminar las barreras al libre flujo de bienes, servicios y capital entre los países. $\mathrm{L} a$ facilitación del comercio consiste en actividades dirigidas a atenuar las barreras innecesarias al comercio y a la inversión en la región del Asia Pacífico. Éstas incluyen el intercambio de información entre los gobiernos sobre barreras no arancelarias y el desarrollo de maneras de reducirlas, la armonización de los estándares en la región, la estandarización y simplificación de los reglamentos y procedimientos aduaneros y el establecimiento de reglas más transparentes para la inversión. La cooperación económica y técnica es necesaria para lograr resultados en las otras dos áreas.
Debido a sus diferentes características, que incluyen distintos grados o etapas de desarrollo económico alcanzado, diferentes políticas de industrialización y distintas estructuras arancelarias, para nombrar sólo unas pocas, los países miembros de la APEC tienen puntos de vista diferentes y divergentes $\mathrm{e}$ interactúan de distinta manera con relación a varios aspectos de las tres áreas de trabajo mencionadas. Sin embargo, estas diferencias no se suscitan sólo al interior del grupo de la APEC. Incluso dentro del grupo regional de la ASEAN, los países miembros difieren respecto a algunos aspectos de las tres áreas de trabajo de la APEC.

De esta manera, la intención del presente documento es rastrear e identificar dichos puntos de vista y modos de interacción de los países: primero, en el caso de la ASEAN y, segundo, en el caso de la APEC. 
ASEAN: hacia la liberalización del comercio y la cooperación económica

La ASEAN fue creada en 1967 por cinco países: Indonesia, Malaysia, Filipinas, Singapur y Tailandia. Brunei se unió a este grupo en 1984, luego de obtener su independencia. La República Socialista de Vietnam hizo lo propio en 1995. Laos y Myanmar fueron admitidos en julio de 1997. Asi, la ASEAN se ha expandido de ASEAN-5 a ASEAN-9y, eventualmente, será ASEAN-10 con la inclusión de Camboya en el futuro cercano. La formación de la ASEAN se basó sobre todo en la necesidad de seguridad regional y cooperación política. Luego de la Declaración de Bangkok de 1967, y hasta 1972 , la ASEAN tuvo muy poco de cooperación económica, y este periodo se caracterizo por ser la "etapa del conocimiento mutuo" y de la "política del evitamiento". También hubo muy poca cooperación cconómica entre los años 1973 y 1975 , debido a la ausencia de compromiso político. Lo significativo de este periodo fue que los paises miembros pudieron deliberar sobre las técnicas recomendadas por el equipo de las Naciones Unidas. Singapur favoreció la liberalización comercial que había sido sustentada por Tailandia, Malaysia y las Filipinas. Indonesia favoreció el esquema de complementación industrial. El periodo de 1976 a 1992 ha sido muy importante en la historia de la ASEAN, ya que durante estos años se llevaron a cabo cuatro cumbres: Bali (1976), Kuala Lumpur (1977), Manila (1987) y Singapur (1992), lo que condujo a un grado más elevado de compromiso político. La cooperación económica en la industria y el comercio ha recibido alta prioridad en los años noventa en respuesta al creciente proteccionismo de Europa y América del Norte.

\section{Liberalización del comercio}

La idea de la liberalización del comercio y la cooperación económica en la ASEAN comenz 6 con el informe del equipo de las Naciones Unidas, cuyos términos de referencia eran la promoción del libre comercio. El informe de las Naciones Unidas recomendaba un plan de acción integral que consistía en la liberalización del comercio y en la cooperación industrial. La liberalización del comercio puede adoptar un enfoque maximalista, que contempla un área de libre comercio con eliminación total de las barreras arancelarias, o uno minimalista, que involucra un enfoque gradual de liberalización, de producto por producto. Las Naciones Unidas recomendaron el último.

En $1974 \mathrm{cl}$ scñor S. Rajaratnam, ex ministro de Singapur, ponía énfasis en que el comercio era el marco de referencia lógico para las actividades económicas de la ASEAN. Había desacuerdo sobre la velocidad y el método de liberalización del comercio. Singapur apoyaba un área de libre comercio con reducción de aranceles a través de las fronteras, que debía ser implementado a un ritmo más rápido. Filipinas apoyaba esta propuesta; Indonesia y Malaysia, sin embargo, preferían un enfoque cauto de paso por paso. Tailandia era neutral, pero tendía a inclinarse por la primera propuesta.

Luego de la primera reunión cumbre de la ASEAN en 1976, las medidas de liberalización del comercio se implementaron desde 1977 a través de Acuerdos Comerciales Preferenciales (PTA) que otorgaban preferencias arancelarias. Los ítem cubiertos se basaban en ofrecimientos individuales y en negociaciones producto por producto, siguiendo un enfoque 
sectorial. Luego este enfoque se cambió por otro que disponía una reducción arancelaria mínima de $20 \%$ a todas las im. portaciones al interior de la ASEAN. Sin embargo, la implementación de los acuerdos preferenciales no fue muy efectiva y tuvo un impacto mínimo sobre el comercio. Cubría sólo $2 \%$ del comercio al interior de la ASEAN en 1980 y $5 \%$ en 1986. Se otorg6 preferencias arancela. rias sólo a 337 de los 12.783 ftem ofrecidos en la lista de acuerdos preferencia. les de 1987. Esta baja tasa de utilización se atribuyó a varios factores, entre ellos una larga lista de exclusiones, una desagregación demasiado detallada y la inclusión de ítem irrelevantes. Con esta baja tasa de utilización, el intercambio comercial al interior de la ASEAN continuó siendo muy reducido, entre 15 y $20 \%$, sobre todo entre Singapur, Indonesia y Malaysia.

Hubo otros factores responsables del reducido intercambio comercial al interior de la ASEAN. Primero, las importaciones de la ASEAN procedentes de los países desarrollados gozaban de aranceles más bajos, mientras que las importaciones de bienes manufacturados procedentes de la ASEAN enfrentaban aranceles más altos. Segundo, la ASEAN ha dependido de pafses no miembros para el comercio, las inversiones extranjeras directas y la tecnología. Tercero, había desacuerdo sobre la velocidad y el método de liberalización. Durante los años cincuenta y sesenta, los paises de la ASEAN adoptaron la política de sustitución de importaciones que restringía las importaciones mediante altas tasas arancelarias. Las Filipinas tuvo la experiencia más larga de sustitución de importaciones, mientras que Singapur tuvo la más corta. Dado el reducido tamaño de su mercado, la escasez de recursos fi- nancieros, el desempleo masivo y la carencia de empresarios nacionales, Singapur se volvió una economía abierta al final de los años sesenta, a través de una política de industrialización basada fuertemente en inversiones extranjeras directas orientadas a la exportación y con uso intensivo de mano de obra. Sin embargo, desde 1979, Singapur ha dirigido su política de industrialización alentando industrias más intensivas en capital, de mano de obra altamente capacitada, elevada tecnología y alto valor agregado. Desde 1970, habiendo aprendido de Singapur, otros países de la ASEAN han optado por una política de industrialización orientada a las exportaciones y dependiente de las inversiones extranjeras directas.

La necesidad de un mayor intereambio comercial al interior de la ASEAN sc identificó ya en 1967. Durante finales de los ochenta, se discutió nuevamente el asunto del libre comercio, idea propuesta durante la cumbre de Manila de 1987, pero que no tuvo acogida. Sin embargo, el ex primer ministro de Tailandia, Anand Panyarachun, apoyado por el primer ministro de Singapur, Goh Chok Tong, revivió el tema en 1991. Varios sectores realizaron estudios de factibilidad en la economía regional. Los académicos recomendaron usar un enfoque diferente para conceder periodos de gracia a los países que no estaban listos aún para una libera. lización acelerada. El sector privado propuso una regla de contenido ASEAN de $40 \%$. Por el lado gubernamental, se crearon comisiones para considerar la idea y para desarrollar un esquema de trabajo práctico. Se condujo discusiones y deliberaciones a través de sesiones de diálogo formal e informal. Durante la $22^{\mathrm{a}} \mathrm{Re}$ unión de Ministros de Economía de la ASEAN, Indonesia propuso un instrumen- 
to co-mercial conocido como el Arancel Preferencial Efectivo Común, que fue adopta-do durante la reunión cumbre de Singapur en 1992. Los líderes de la ASEAN firmaron una declaración que dispone la creación de una Zona de Libre Comercio de la ASEAN para el año 2008, un periodo de 15 años. Sin embargo, con un compromiso político mayor $y$ en consonancia con los acontecimientos mundiales, los lifderes de la ASEAN acordaron llegar a la AFTA más bien en el año 2003, cinco años antes que la meta inicialmente propuesta.

La AFTA trae consigo una serie de beneficios. Primero, crea mayor intercambio comercial al interior de la ASEAN. Segundo, originará un flujo libre de bienes y servicios al interior de esta región; con alrededor de 500 millones de personas, crecerán la producción y el consumo. Tercero, generará cconomías de escala. debido a que la producción se basará en ventajas comparativas. A su vez, esto redundará en mayor especialización, incremento de la escala de la producción, reducción de los costos de producción y precios más bajos. Cuarto, la AFTA atraerá más inversiones extranjeras directas de corporaciones transnacionales, particularmente de aquellas relacionadas con el comercio.

Alcanzar la AFTA no estará libre de problemas. Primero, hay ausencia de compromiso por parte del sector privado. Segundo, se generará desempleo por las industrias ineficientes. Tercero, los estándares técnicos y de seguridad no están armonizados, tampoco los reglamentos de despacho de aduanas. Cuarto, las estructuras arancelarias difieren; Indonesia, $\mathrm{Fi}$ lipinas y Tailandia cuentan con los aranceles más altos. Una reducción de los a- ranceles ocasionaría pérdidas de ingresos.

Para llegar a la AFTA, desde 1993 se ha implementado un instrumento conocido como el Arancel Preferencial Efectivo Común, que se aplica a manufacturas, bienes de capital y productos agrícolas procesados. Todos estos productos se han clasificado en dos grandes grupos: "bienes prioritarios" y "otros bienes". Los aranceles a los bienes prioritarios deberian reducirse a entre 0 y $5 \%$ y a un ritmo más rápido que los aranceles para los otros bienes. El objetivo final es alcanzar aranceles de entre 0 y $5 \%$ para todos los bienes en el año 2003. A los nuevos miembros de la ASEAN se les otorga un plazo adicional, hasta el año 2006 en el caso de Vietnam, y hasta el 2008 en los casos de Laos y Myanmar. Los 15 productos prioritarios incluidos en el esquema acelerado son: pulpa, cemento, fertilizantes, aceite vegetal, gemas y joyería, productos de caucho, productos farmacéuticos, cátodos de cobre, productos de cuero, muebles de madera y de mimbre, plásticos, textiles, químicos, electrónicos y productos de cerámica y de vidrio.

Las medidas de liberalización tomadas desde 1993 han probado ser efectivas, como lo indica la tendencia ascendente del intercambio comercial al interior de la ASEAN. Éste creció significativamente en aproximadamente $40 \%$, pasando de 79 mil millones de dólares en 1993 a 111 mil millones de dólares en 1994. En 1995, se incrementó hasta 140 mil millones de dólares, lo que corresponde al $25 \%$ del comercio global de la ASEAN. La adopción, por parte de las economías de la ASEAN, de políticas comerciales orientadas al exterior y de políticas de tasas de cambio pragmáticas, combinada con la rápida expansión de las exportaciones, 
indica que la ASEAN está siguiendo el mismo camino que los países recientemente industrializados (NIC) transitaron en los años sesenta y setenta.

\section{Cooperación industrial}

En el área de la cooperación económica, los esfuerzos de la ASEAN probaron tener resultados variables. Los primeros esquemas de cooperación industrial comprendieron los Proyectos Industriales, la Complementación Industrial y los Joint Ventures Industriales.

El equipo de las Naciones Unidas propuso el esquema de los proyectos industriales en 1973, el que se adoptó oficialmente en 1976 y se ratificó en 1980. Este esquema tenía por objetivo conseguir algunos beneficios, entre ellos, menor dependencia de fuentes extranjeras de bienes importados, mejora del comercio al interior de la ASEAN, creación de empleo y contribución a la producción de bienes alimenticios y a las exportaciones. El primer paquete de proyectos industriales concebido se asigno así: el proyecto de fertilizantes de úrea, a Malaysia e Indonesia; el de motores diesel, a Singapur; el de superfosfatos, a Las Filipinas, y el de carbonato sódico, a Tailandia. Esta distribución dependió de la abundancia de materias primas: petróleo y gas en Malaysia e Indonesia, cobre y ácido sulfúrico en Las Filipinas y vastos depósitos de sal en Tailandia. Singapur está capacitado para fabricar pequeños motores debido a sus siderúrgicas, a su infraestructura bien desarrollada para importar insumos necesarios y exportar productos finales, y a sus trabajadores altamente calificados. Sin embargo, de los cuatro proyectos de la ASEAN sólo están operativos los proyec- tos de fertilizantes de úrea de Indonesia y Malaysia. Singapur canceló el suyo debido a cambios en su economía e Indonesia presentó cierta resistencia. Las Filipinas disminuyó su producción debido a la caída de los precios del cobre. Tailandia se retiró debido a la cafda de los precios del carbonato sódico. En general, el esquema de los proyectos industriales no tuvo éxito, pues no hubo estudios de factibilidad y se trata de proyectos industriales regionales de gran escala que requieren una enorme financiación: entre 300 y 400 millones de dólares a precios de 1976.

El esquema de complementación industrial fue propuesto primero en 1976 , y el acuerdo respectivo se firm6 en 1981 . Se basaba en sumar recursos y en compartir mercados. Los dos paquetes propuestos en 1976 implicaban la producción y distribución de partes y componentes automotrices. El primer paquete se implementó a partir de 1983, mientras el segundo se pospuso indefinidamente, ya que los países miembros no se pusieron de acuerdo respecto de la asignación de productos. Surgieron también otros problemas, comola falta de apoyodel mercado $y$ las reducciones arancelarias, $y$ diferencias en las marcas y los tipos de vehículos. El primer esquema de complementación industrial contribuyó con menos del $1 \%$ al intercambio comercial al interior de la ASEAN durante el periodo comprendido entre 1982 y 1985. El segundo esquema de este tipo en la industria automotriz se conoció como la Complementación Marca a Marca e involucró el intercambio de productos. Hubo preferencia por las partes que contenían gran valor agregado, como la fabricación de motores. No hubo problemas de especificaciones, puesto que se trataba de la misma marca. Entre los países de la ASEAN, Singapur y Brunei no 
participaron por no contar con industrias automotrices. Indonesia tampoco lo hizo pues quería proteger su propia industria y mercado. La primera empresa en usar este esquema fue Mitsubishi en Manila. A ésta siguieron Volvo, Mercedes Benz, Nissan, Toyota, el grupo DAF de Bélgica $y$ Renault en Tailandia y Malaysia. Indonesia mostró un interés creciente en el esquema a medida que algunas compañfas japonesas reubicaban parte de sus procesos de producción en su territorio debido a los crecientes costos en el Japón. En 1991, dado que este acuerdo de Complementación Marca a Marca alcanzó mejores oportunidades de éxito, el acuerdo se amplí a productos no automotrices. Actualmente se encuentran operativos alrededor de 33 proyectos de este tipo, que involucran a mís de 10 fabricantes de vehículos.

El esquema de las joint ventures industriales fue propuesto por el sector privado en 1980, y el acuerdo correspondiente se firm6 en 1983. Apuntaba a promover las inversiones entre inversionistas privados y a incrementar la producción industrial en la región a través de sumar recursos y compartir mercados. Los productos de este esquema recibieron también mayor tratamientopreferencial. Las jointventures industriales fueron más flexibles $\mathrm{y}$ alcanzaron mayor mérito que los anteriores esquemas porque no requerían que su propiedad fuera íntegramente de miembros de la ASEAN. La propiedad del capital de la ASEAN se redujo a $40 \%$ en 1987 ; en la propiedad de estas empresas pueden participar ciudadanos de países no miembros. La mayoría de joint ventures industriales tiene participación de capitales extranjeros.

Las recientes iniciativas para promo- ver la cooperación industrial en la región de la ASEAN adoptan la forma de lo que se conoce como el esquema de Cooperación Industrial de la ASEAN, que apunta a incrementar la producción industrial de la región y las inversiones, tanto de fuentes de la ASEAN como de fuentes de países no micmbros, a generar industrias propias de la región que sean competitivas internacionalmente, a fomentar la participación del sector privado y a incrementar la complementación industrial. Este esquema fue propuesto por el sector industrial y el acuerdo respectivo se firmó en abril de 1996. Reemplaza a los esquemas de complementación de marca a marca y de joint ventures industriales y se encuentra operativo desde noviembre de 1996. Este esquema apunta a satisfacer las necesidades de cmpresas pequeñas y medianas. Comprende al menos dos compañías de dos países diferentes, las cuales deben tener por lo menos $30 \%$ de capital nacional. Dentro de este esquema caben productos finales, intermedios o materias primas, los que gozan de aranceles preferenciales de entre 0 y $5 \%$, previa aprobación. La Cooperación Industrial de la ASEAN adopta varias modalidades: joint ventures, fabricación conjunta, transferencia de tecnología, capacitación, licencias, compras y adquisiciones consolidadas, servicios gerenciales y servicios de ventas y mercadeo.

\section{APEC: Hacia la liberalización comer- cial y la cooperación económica}

La idea de formar un grupo regional para la Cooperación Económica Asia Pacífico (APEC) surgió debido al proteccionismo creciente en Europa y Norteamérica y a la incertidumbre reinante en las negociaciones comerciales multilaterales origi- 
nadas por el lento progreso de la Ronda de Uruguay.

La APEC comprende diferentes tipos de economías de la región de Australasia, el Sudeste y el Este de Asia, el Norte y el Sur de América. La primera reunión de APEC fue en Canberra, Australia, en 1989 , y a ésta asistieron 12 países miembros, incluyendo a 6 miembros de la ASEAN: Brunei, Indonesia, Malaysia, Las Filipinas, Singapur y Tailandia; así como países de la OCDE, como Japón, Estados Unidos, Canadá, Australia, Nueva Zelanda y Corea del Sur. La APEC ha progresado rápidamente desde 1989 incrementando el número de sus miembros a 18 paises. China, Taiwan y Hong Kong se incorporaron en 1991, Papúa Nueva Guinea y México lo hicieron en 1993 y Chile lo hizo en 1994. Otros países han expresado interés en unirse a la APEC, como Corea del Norte, India, Vietnam, la Federación Rusa, Perú y Colombia.

A fines de los años ochenta y principios de los noventa, las negociaciones de la Ronda de Uruguay parecían haber quedado empantanadas. Estados Unidos contempló una opción de repliegue bajo la forma de un acuerdo comercial regional. $\mathrm{Se}$ consideraba que un enfoque regional antes que uno global traería la liberalización comercial y de inversiones. En 1988, Estados Unidos firmó su acuerdo de libre comercio con Canadá. El Acuerdo de Libre Comercio Norteamericano, o NAFTA, se firmó junto con México en 1994. Todas las barreras al movimiento de bienes, servicios y capitales entre los países miembros se eliminarán en un plazo de entre 10 y 15 años por medio de este acuerdo. Posteriormente ha habido discusiones sobre la ampliación del acuerdo para incluir a América Central y América del Sur.
Para impulsar un acuerdo de libre comercio con la región asiática, Estados Unidos, con el apoyo entusiasta de Australia, se interesó seriamente en la APEC iniciada en Australia. El Grupo de Personas Eminentes, liderado por el profesor Fred Bergsten, ha sido un elemento clave, pues las recomendaciones del grupo eran compatibles y consistentes con los planes de los Estados Unidos. El fuerte impulso de este último país para lograr el éxito de la APEC provecrá un poder de negociación incrementado cuando se compita con la Unión Europea.

\section{Área de atención preferencial}

Los países miembros de la APEC tienen puntos de vista diferentes y divergentes sobre algunos aspectos de las tres áreas de trabajo. Países miembros como Estados Unidos, Australia, los países de la ASEAN, Japón y China han expresado algún tipo de desacuerdo en una serie de asuntos. El primero se refiere al área a la cual darle la mayor atención. De las tres áreas de trabajo de la APEC, el interés de Estados Unidos se centra en que la APEC actúe como un mecanismo para la liberalización del comercio y la inversión. Este país se opone al fuerte interés que pone la APEC en la cooperación económica y se ha mostrado indiferente respecto de la facilitación del comercio. Por otro lado, Japón pone el énfasis en la cooperación económica. La actitud de Japón en la APEC es comprensible, pues ya ha integrado las economías de Asia desde 1960 por un acuerdo comercial formal a través de inversiones, comercio, asistencia para el desarrollo y tecnología japoneses en Asia, particularmente en la ASEAN. Japon ha creado un mercado cautivo en la región de Asia Pacífico. 


\section{¿Cómo enfocar la APEC?}

El segundo aspecto en el que existe desacuerdo es en términos de cómo enfocar la APEC. Estados Unidos quiere un enfoque legalista e institucional, mientras que los gobiernos de Asia abogan por un enfoque evolucionista al estilo consensual de la ASEAN. Más aún, Estados Unidos desca fijar un plazo. Los estadounidenses resultaron frustrados debido a la falta de entusiasmo y apoyo del Japón y otros países asiáticos. Japón se opone a la idea de liberalizar completamente el comercio para el año 2020; querría, por ejemplo, eximir al sector agrícola japonés del plazo del 2020. Australia y Estados Unidos se opusieron fuertemente a la posición de Japón.

\section{¿Es la APEC otro bloque comercial?}

El tercer aspecto del desacuerdo estriba en que la APEC se esté convirtiendo en otro bloque comercial. Estados Unidos y Australiaestán empeñados en que la APEC se convierta en un acuerdo de libre comercio con todas las de la ley. Sin embargo, la mayoría de miembros asiáticos fueron cautos y no expresaron abiertamente su resentimiento para no antagonizar con Estados Unidos.

Malaysia es ciertamente una excepción. El doctor Mahathir, quien boicoteó la cumbre de los líderes de la APEC en Seattle en 1993, declaró en setiembre de 1994 que la APEC no debía convertirse en una comunidad estructurada ni tampoco en un bloque comercial. Más aun, que no deberfa opacar totalmente a la ASEAN o ser dirigida por miembros más poderosos. Puso énfasis en la igualdad entre todos los países miembros de la APEC.

\section{¿Debería haber fronteras?}

El cuarto aspecto del desacuerdo reside en las fronteras geográficas. Como Estados Unidos no quiere estar excluido de los mercados asiáticos, se opone a la idea de tener un bloque comercial asiático dominado por el Japón en un lado del Pacífico y un bloque norteamericano en el otro lado. Sin embargo, Malaysia ha propuesto la formación de un Grupo Económico del Este de Asia (EAEG) formado por países del Este y del Sudeste de Asia con Japón en el centro. Este grupo excluye a Australia y a Estados Unidos. Otros gobiernos del Sudeste de Asia apoyaron la propuesta de Malaysia; sin embargo, China, Corea y Japón no lo hicieron, y favorecieron, por el contrario, a la APEC. Estados Unidos y Australia presionaron fuertemente a los países miembros de la APEC para que en la reunión de la APEC de 1994 en Indonesia se determinara que la EAEG fuese denominada Comité EAE, la misma que se abrevia EAEC (por sus siglas en inglés), que se constituiría en un cuerpo independiente dentro de la APEC.

En general, Estados Unidos quiere ver planes de acción definidos con el fin de lograr la liberalización del comercio en el 2010 para los países desarrollados y en el 2020 para los países en desarrollo. Sin embargo, no todos los estadounidenses apoyan la idea de un área de libre comercio en la región Asia Pacífico. Los académicos y hombres de negocios ven la agrupación económica como irreal dada la amplia y diversa gama de sus miembros. También hay muchos estadounidenses que están en contra de la idea de establecer un primer plazo (2010) para que Estados Unidos liberalice su comercio y un plazo posterior (2020) para que lo ha- 
gan los países en desarrollo. Arguyen que los Estados Unidos estarán en desventaja. Las exportaciones de los países en desarrollo no enfrentarán aranceles en los mercados estadounidenses, mientras que las de Estados Unidos sí tendrán que pagar aranceles en esos países.

\section{Regionalismo abierto}

El quinto aspecto del desacuerdo es el contexto de regionalismo abierto. Para Estados Unidos el regionalismo abierto significaría que el arreglo estaría abierto para todos los países miembros que cumplan con los acuerdos, y se discriminaría a todos los países no miembros, aunque sea transitoriamente. El punto de vista estadounidense apunta a evitar que los no miembros aprovechen la circunstancia. Sin embargo, el punto de vista asiático es promocionar un regionalismo abierto que no implique la discriminación de los no miembros.

\section{La ASEAN y la APEC hacia la con-} vergencia: influencia en la región

El 20 de noviembre de 1993, los líderes económicos de la APEC, recibidos por el presidente Clinton, se reunieron en Blake Island en Seattle, Washington, para llevar a cabo discusiones informales. Los líderes económicos preveían una reducción continua de las barreras comerciales y de las barreras a la inversión para expandir el comercio en la región del Asia Pacífico $y$ con el mundo, y para que los bienes, servicios, capital e inversión circularan libremente entre las economías de la APEC.

En la Declaración de Bogor de 1994, las economías de la APEC se comprome- ten a alcanzar la liberalización y la apertura del comercio y las inversiones en la región del Asia Pacifico, en el 2010 para las economías industrializadas y en el 2020 para las economías en desarrollo. Las economías de la APEC se comprometen también a implementar los resultados de la Ronda de Uruguay de 1993 para apoyar activamente el sistema comercial multilateral, para continuar con la liberalización unilateral del comercio e inversiones y para evitar imponer nuevas medidas proteccionistas.

En 1995, los líderes económicos de la APEC adoptaron el Plan de Acción de Osaka, que sirve como patrón de referencia para implementar su compromiso con el comercio e inversión libres y abiertos, la facilitación comercial y la cooperación téenica y económica en las áreas de energía y transporte, infraestructura, pequeña y mediana empresa y tecnología agrícola.

En 1996, los líderes económicos de la APEC adoptaron el Plan de Acción de Manilapara la APEC(MAPA), que consiste en planes de acción individuales y colectivos $\mathrm{e}$ informes de avance sobre actividades conjuntas para cumplir con los objetivos de Bogor y de Osaka. MAPA gira alrededor de seis temas: mayor acceso al mercado de bienes, acceso ampliado al mercado de servicios, régimen de inversión abierto, costos reducidos en los negocios, sector de infraestructura abierto y eficiente y cooperación técnica y económica fortalecida.

Además de las reuniones de Bogor y Subic Bay, la región de ASEAN acogió también otras dos reuniones internacionales significativas en 1996, la reunión AsiaEuropa (ASEM) en Bangkok y la reunión de la Organización Mundial de Comercio 
en Singapur. Esto indica la creciente importancia y el apoyo de la región de la ASEAN a las actividades económicas de la APEC. A su vez, las economías de la APEC han desempeñado, y continuarán haciéndolo, un papel crucial en el desarrollo económico de esta región, ya que resulta económicamente importante en términos de flujos de inversión y comercio, y también en términos de su contribución a la producción mundial, además del enorme tamaño de su mercado. La APEC representa un PNB acumulado de alrededor de 13 billones de dólares (aproximadamente la mitad de la producción mundial total) y una población cercana al $40 \%$ del total mundial.

La APEC y la ASEAN deberían complementarse entre sí, en vez de competir. Esto será posible sólo si la APEC permanece como una organización de estructura abierta. Más aún, la APEC puede complementar a la ASEAN si practica el regionalismo abierto para promover el multilateralismo. Así, tanto la APEC como la ASEAN, a través del AFTA, tienen papeles cruciales que desempeñar en la promoción de la liberalización comercial.

La cuestión es cómo la APEC puede emerger como líder en el libre comercio global. Los miembros de la APEC deberían comprometerse con el establecimiento del libre comercio. La ASEAN debería encabezar el movimiento a medida que logre un área de libre comercio para el 2003. Puede trasladar las reformas de libre comercio hacia la región más grande de la APEC y luego a otras regiones en el mundo. La APEC también ha mostrado su liderazgo potencial en la reciente reunión de la Organización Mundial de Comercio en Singapur.

\section{Conclusión}

Tanto dentro del bloque regional de la ASEAN como dentro del foro de la APEC, los países miembros tienen puntos de vista diferentes e interactúan de distinta manera. Dentro de la ASEAN, los países miembros difieren en la velocidad del proceso de liberalización del comercio y en la asig. nación de proyectos industriales en la búsqueda de la cooperación industrial. Dentro de la APEC, los países miembros tienen puntos de vista diferentes $\mathrm{e}$ interactúan de distinta manera en términos de enfoque, plazo, asuntos de bloqueo comercial y regionalismo abierto. Pero ambas, tanto la ASEAN como la APEC han mostrado convergencia de intereses acerca de la necesidad de lograr la liberalización y la apertura del comercio y las inversiones. Tanto la APEC como la ASEAN tienen papeles cruciales que desempeñar para lograr el comercio global. 


\section{Referencias Bibliográficas}

ATKINSON, Jeff. APEC: Winners and Losers. Australia, Australian Council for Overseas Aid, 1995. Background Report N ${ }^{\circ}$ 7.

BUREAU OF INDUSTRY ECONOMICS. Potential Gains to Australia from APEC: Open Regionalism and the Bogor Declaration. Australia, 1995. Occasional Paper 29.

CHIA SUOW, Yue. APEC Challenges and Opportunities. Singapur, Institute of Southeast Asian Studies, 1997.

CHIA SUOW, Yue and TAN, Joseph. ASEAN in the WTO. Singapur, Institute of Southeast Asian Studies, 1996.

DAQUILA, Teófilo C. "The ASEAN Región's Role in the Future Global Economy". En Business Briefing, ASEAN at 30. London, World Market Research Centre, ASEAN Secretariaty US-ASEAN Council, 1997, p. 38-41.

SURIYAMONGKOL, Marjorie. Politics of ASEAN Economic Cooperation. Singapur, The Institute of Southeast Asian Studies y Oxford University Press, 1988. 